\title{
La Actividad Turística: diagnósticos micro y macroeconómicos
}

The Tourist Activity: micro and macroeconomics diagnoses

Alfredo Ascanio ${ }^{1}$

RESUMEN: El objetivo de este artículo es proporcionar a los estudiantes de turismo una herramienta de trabajo para realizar diagnósticos microeconómicos de empresas turísticas y de esa manera evaluar las interrelaciones de aquellos factores críticos que puedan estar limitando la efectividad de las operaciones y por tanto disminuyendo la productividad del negocio; igualmente, se discute la herramienta de Insumo-Producto, como una manera de diagnosticar las posibles bondades del sector turismo, a fin de planificar mejor y establecer políticas adecuadas. El artículo se basa en la literatura denominada Análisis de Factores, producida en las décadas de 1950 y 1960 por el economista austríaco dr. Alfred Klein, asesor de Naciones Unidas; y en los esfuerzos del Instituto de Estudios Turísticos español, para generar tablas de insumo-producto, durante la década de 1970. De esta manera no se descuidan los aportes clásicos de esos años y se rescatan metodologías útiles para el diagnóstico.

PALABRAS CLAVE: diagnóstico de empresas; matriz de insumoproducto.

ABSTRACT: The objective of this article is to provide the students of tourism a tool of work to carry out microeconomic diagnoses of tourist businesses

1. Economista y Ph.D. en Ciencia Política. Profesor titular jubilado del Departamento de Ciencias Económicas y Administrativas de la Universidad Simón Bolívar. Autor de al menos treinta artículos arbitrados en tres revistas internacionales de turismo. Ha publicado dos libros sobre turismo: Elementos del turismo como sistema (1998), Turismo e planejamento hoteleiro (2003) y un libro sobre ciencia política: Análisis de contenido del discurso político (2001). Contacto: Urb. Alto Prado, Calle 10 -Qta. Los Helechos - Caracas 1080, Venezuela; e-mail: ajascanio@cantv.net. 
and of that way to evaluate the interrelations of those critical factors that may be limiting the effectiveness of the operations and therefore diminishing the productivity of the business; likewise, the tool of input-output is discussed like a way of diagnosing the possible kindnesses of the Tourism and to establish adequate politics. The article is based on the literature called Análisis of Factors, produced in the decade of the years 50 and 60 by the austrian economist dr. Alfred Klein, advisor of United Nations; and in the efforts of the Institute of Spanish Tourist Studies, to generate boards of imput-output during the decade of the years 70 . In this manner themselves them they are not neglected contribute classics of those years and useful methodologies for the diagnostic.

KEYWORDS: diagnosis of businesses; input-output analysis.

\section{Introducción}

En la actividad turística han surgido algunas brechas entre la enseñanza y la investigación. Algunos textos docentes hacen más énfasis en los aspectos conceptuales y en las definiciones y mucho menos en la metodología para investigar y en especial para diagnosticar y evaluar. No obstante, la mayoría de las revistas dedicadas al turismo sí acentúan el uso de las técnicas de análisis, con mayor o menor grado de rigurosidad.

Este artículo, denominado diagnósticos micros y macros, es el producto de varios cursos experimentales que se han desarrollado con la intención de reducir esa brecha. Se puede decir que debido a la existencia del libro de Stephen L. J. Smith, titulado Tourism analysis: a handbook (1989), y las publicaciones de Regina Schlüter: Investigación en turismo y hotelería (2000); de Mirian Rejowski: Turismo e pesquisa científica (1996); de Margarita Barretto: Manual de iniciação ao estudo do turismo (1995); y de los editores J. R. Brent Ritchie y Charles R. Goeldner: Travel, tourism, and hospitality research, ya no hace falta insistir en las herramientas más importantes para la investigación en turismo. Lo que si es posible explicar, con algún detalle, es la metodología que se puede utilizar para hacer primero el diagnóstico de la empresa turística, ya que se podría partir de allí, para entonces aplicar otras técnicas más sofisticadas que permitan tomar adecuadas decisiones. Igualmente, se trata de promover de nuevo las posibilidades del uso de la matriz de Insumo-Producto, a los fines de planificar mejor el sector y generar políticas adecuadas.

El artículo se basa en la metodología divulgada por el economista austríaco Alfred Klein, quién trabajó tanto en México como en Venezuela, en el área de la productividad y el diagnóstico de empresas. Klein escribió tres obras de importancia fundamental, que han servido como bibliografía básica para el artículo; o sea, primero escribió en México, con la colaboración de Nathan Grabinsky, el libro El análisis factorial: una guía para estudios de economía industrial (1958); luego a esta obra siguió La productividad como criterio de inversión: una guía para evaluación de proyectos industriales (1961). Estos dos libros se complementan con una guía publicada en Venezuela, por el Instituto Venezolano de Productividad y titulada El diagnóstico de empresas (1966).

Así pues, esta herramienta metodológica se complementa con las otras que se encuentran en los libros de Manuel Figuerola Palomo, titulados Elementos para el estudio de las economía de la empresa turística (1990) y Economía para la gestión de las empresas turísticas (1995); e igualmente, metodologías aportadas en la obra de Mário Carlos Beni, Análise estrutural do turismo (1997).

En este contexto es útil revisar, de nuevo, los aportes que sobre las tablas de Insumo-Producto realizaron en España los investigadores Ángel Alcaide, Valentín Andrés Álvarez, Manuel de Torres, José Largo, Antonio Pulido San Román, Manuel Figuerola e Ignacio de Cuadra, pioneros en los análisis macroeconómicos del turismo español.

\section{Diagnóstico para administrar}

Diagnosticar consiste en determinar los síntomas y el carácter de un asunto. Se dice de los signos que permiten reconocer un problema, con el fin de llegar luego a una posible solución. Toda empresa, como negocio, dispone de recursos y con ellos realiza una actividad operativa para generar beneficios. La idea central es que seguramente deben existir características comunes a toda empresa, de manera que se pueda desarrollar una metodología para el análisis que permita conocer cómo los factores de una empresa forman un sistema; y darnos cuenta, que si surge un área crítica, en cualquiera de sus factores, ello limitará la productividad del conjunto, si oportunamente no se corrigen las fallas.

La persona que administra una empresa turística, por ejemplo, debe tener la experticia para dirigir y controlar sus actividades. Muchas veces, el peligro de administrar consiste en caer en métodos rutinarios y desentenderse de las previsiones que se deben tomar, si cambia el contexto. Anticiparse a los posibles problemas futuros es mucho mejor que ignorarlos. 


\section{Factores para el diagnóstico}

Toda empresa, y la turística no escapa a esto, se ve afectada a menudo por el ambiente como son las decisiones de la clientela, las fuerzas competitivas de empresas iguales o similares y el desarrollo tecnológico, entre otros. El ambiente le crea a la empresa muchos riesgos, pero también le ofrece muchas oportunidades. Es necesario entonces diagnosticar el ambiente, como marco general exógeno, antes de hacer cualquier diagnóstico en el interior de la empresa misma.

La empresa tiene que establecer un objetivo global, que luego debe segmentar en objetivos departamentales, según su contribución al éxito que se ha establecido como meta. La función de la gerencia consiste, justamente, en establecer la misión, visión y el objetivo general de la empresa; y en base a ese objetivo, definir las políticas y dirección.

Toda empresa se dedica a producir bienes y servicios para una determinada clientela. El diseño de esos productos y servicios y los métodos para producirlos, dependen del mercado, de la tecnología y otros factores. Es relevante conocer los productos y los procesos de Producción, para poder realizar un adecuado diagnóstico.

Las políticas financieras de la empresa son decisivas. Administrar bien los ingresos y costos y la manera de financiar las operaciones de la empresa es un asunto relevante. El capital es un bien tan escaso, que es necesario tratar de reducirlo por unidad de producción conocida, ya que el costo financiero puede ser elevado.

Las inversiones en medios de producción y la intensidad de los capitales invertidos en construcciones, maquinarias y equipos, así como su mantenimiento y sustitución a tiempo, es un tema que se vincula mucho con la posible productividad.

El éxito de la empresa moderna es su personal capacitado para ejercer sus funciones. La fuerza de trabajo es quizá más importante que otros factores, porque es ella la que lleva a cabo toda la actividad productora.

Los materiales y servicios comprados a otras empresas para obtener un buen aprovisionamiento y lograr economías de compras, es un factor que se relaciona con los proveedores y con los almacenes para mantener las existencias.

La producción es la clave, pues la empresa existe para producir con elevada productividad y obtener beneficios, a fin de crecer y desarrollarse. El plan de producción según los procesos y operaciones, el control de la calidad y las normas para controlar la productividad, son asuntos básicos al realizar el diagnóstico.

Toda empresa tiene que mercadear sus productos y servicios. Es decir, no se produce sólo para almacenar, sino para distribuir y vender en los mercados, a través de la promoción, la publicidad y los contactos con los canales de ventas.
Para realizar todas sus operaciones, la empresa necesita de una política de controles y para ello requiere de estadísticas, de contabilidad y de una realimentación constante de información sobre sus funciones internas y de lo que estaría pasando en su ambiente.

En este apartado hemos colocado en mayúsculas los principales factores que se necesitan diagnosticar, con la idea básica de obtener una adecuada productividad, una buena economicidad; y luego, lograr la rentabilidad que se espera obtener, según la naturaleza de cada negocio.

En seguida aparece un gráfico (Figura 1) sobre la estructura funcional de una empresa, el cual recoge todos los factores que deben ser considerados para realizar un adecuado diagnóstico.

\section{EL ANÁLISIS FACTORIAL}

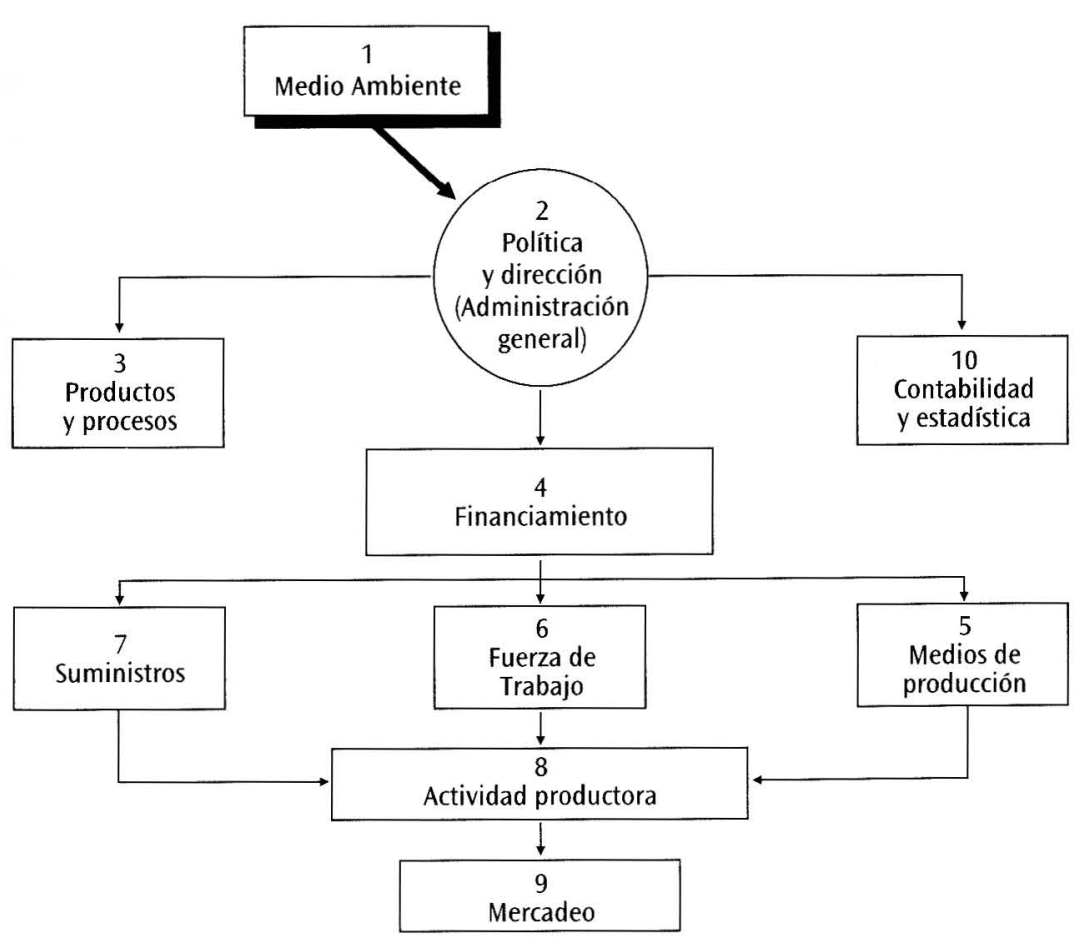

Figura 1. Gráfico - Estructura funcional de la empresa según su operación 


\section{El ambiente: primer factor para el diagnóstico}

Al factor ambiente se le puede asignar la siguiente función: "mantener oportunamente informada a la empresa turística sobre los cambios que ocurren en el contexto que le pertenece e informar igualmente a su clientela y proveedores acerca de sus actividades recientes" (Klein, 1958).

Este factor tiene al menos cuatro elementos. Por ejemplo, el elemento fisico debe ser investigado en lo que se refiere a la localización de la empresa turística, ya que en la medida en que su ubicación sea cercana a sus mercados estratégicos y a sus proveedores de servicios en espacios geográficos especiales, en esa misma medida la empresa puede captar una buena clientela a menores costos y generar volúmenes de ventas convenientes; además, si sus socios están en los mismos lugares para realizar el mercadeo de sus paquetes, ello asegura su venta oportuna. No obstante, hoy existe una localización virtual en Internet, que puede generar buenos negocios. El elemento político se refiere al marco legal para el fomento o la restricción de las operaciones empresariales. El elemento económico tiene que ver, al menos, con cuatro componentes, como son: el mercado, las posibilidades de financiamiento, las tasas impositivas y las actitudes de la sociedad hacia el fomento del turismo. Finalmente el elemento social, tiene como componente las habilidades y adiestramientos necesarios para cumplir la misión y las metas, así como las relaciones humanas.

Ahora bien, metodológicamente es necesario preparar un cuestionario con las preguntas pertinentes, para conocer si se cumple en forma óptima la función del ambiente. Las preguntas se deben hacer siempre en base a los cuatro elementos principales; y en relación también a los valores promedios de las empresas locales de igual o similar tamaño.

\section{Política y dirección: el segundo factor para el análisis}

Al factor política y dirección se le puede asignar la siguiente función: "fijar a la empresa una misión y unos objetivos razonables y proveerla de los medios necesarios para alcanzarlos de manera económica" (Klein, 1958).

El factor tiene al menos tres elementos, como son: política de la empresa con los siguientes componentes: política en base a la clase de actividad y su esfera de actuación; es decir, si la empresa ha definido o no satisfactoriamente la clase de actividad que desea realizar, el alcance y el objetivo básico. Otro componente se refiere a las razones para decidir sus actividades, pues esto puede surgir: bien sea porque los propietarios han tenido una experiencia previa, porque el lugar de ubicación indica que esa debe ser la tradición de sus actividades; o bien, porque hay ventajas para vender, abastecerse de recursos y del financiamiento. Otro componente se refiere a la política de los recursos a utilizar y a los objetivos relacionados con los servicios a su clientela; la manera de añadir valor y aumentar los ingresos, tomando en consideración a los propietarios, los trabajadores y la comunidad. Otro asunto a indagar se refiere a la organización de la empresa para dirigir y controlar sus operaciones, buscando o no un equilibrio y la cooperación de todos los empleados y actores involucrados.

Para realizar el diagnóstico de este factor, no basta con la preparación de un cuestionario, que sería la herramienta cualitativa a utilizar. Será necesario también estimar algunos ratios o relaciones financieras, de productividad y otros indicadores, para medir cuantitativamente la ejecución de la función del factor.

Todos estos ratios o relaciones se obtienen de los Estados de Pérdidas y Ganancias y del Balance General de la Empresa. Por ejemplo, un conjunto de indicadores y sus respectivos pesos, permitiría obtener un Índice General de la Gestión Financiera - IGGF, como sigue:

\begin{tabular}{|l|c|}
\hline - Activo circulante/obligaciones a corto plazo & $25 \%$ de peso \\
\hline - Capital contable/activo fijo & $15 \%$ de peso \\
\hline - Capital contable/obligaciones totales & $25 \%$ de peso \\
\hline - Ventas netas/cuentas por cobrar & $10 \%$ de peso \\
\hline - Ventas netas/inventarios & $10 \%$ de peso \\
\hline - Ventas netas/activo fijo & $10 \%$ de peso \\
\hline - Ventas netas/capital contable & $5 \%$ de peso \\
\hline
\end{tabular}

El índice de productividad se estima como sigue: Producción en términos físicos como el número de pernoctaciones en un año dado, o el número de cubiertos vendidos o el número de paquetes de excursiones vendidos, o el número de turistas movilizados, es decir: cualquiera de estos datos forman el numerador del índice. El denominador estaría formado por la suma del total de horas-hombre utilizadas para realizar la actividad, más la relación entre el costo de los salarios para producir el servicio, entre el promedio del salario por hora de servicio.

Otro indicador se refiere al llamado punto de equilibrio, en términos de producción, es decir: los costos fijos/1 - costos variables/ventas.

Otras relaciones tienen que ver con el rendimiento sobre ventas, sobre patrimonio o capital social; y además, el rendimiento de todo el capital contable invertido; y las ventas netas, entre el número de trabajadores empleados, así como entre el activo total utilizado. 


\section{Productos y procesos: el tercer factor para el análisis}

Al factor productos y procesos se le puede asignar la siguiente función: "seleccionar para la producción de los servicios los atractivos que al mismo tiempo que pueden satisfacer al cliente, rindan beneficios a la empresa y a la comunidad receptora; y así mismo determinar los procesos adecuados para que se puedan ofertar servicios de calidad" (Klein, 1958).

El factor tiene al menos tres elementos, como son: productos y servicios con los siguientes componentes: política de producción, clase de productos y servicios, cantidad del valor de las ventas a precios de costos y el costo unitario por servicio proporcionado. Luego los precios de venta a los Tour Operadores, a los Agentes de Viajes y finalmente a los turistas o clientes. Características de los procesos y el grado de valor añadido, para que los servicios sean atractivos. Mejora de los servicios y procesos para ofertarlos.

Para diagnosticar este factor y sus elementos, recurrimos al cuestionario de preguntas para conocer si existe una definición adecuada de cada producto y servicio, según sus características y calidad que deben alcanzar, sus precios, el tamaño que se desea colocar y la presentación al cliente. ¿¿Cuáles son los cinco principales productos competidores que pueden ser superiores a los de la empresa en calidad, precio y en estilo de los procesos ofertados, incluyendo el valor añadido para lograr una satisfacción psicológica? ¿Cuáles son las principales quejas detectadas? ¿Cuáles servicios están aumentando o disminuyendo en los últimos años? ¿Cuáles son las mejoras de la calidad que se han logrado; y cómo se mejora la producción? ¿Qué medios se emplean para disminuir costos y ajustarse a las características de la comunidad de acogida? Hay más preguntas que se pueden realizar para conocer los aciertos o las fallas de este factor y ello depende de cada tipo de diagnóstico.

\section{Financiamiento: el cuarto factor para el análisis}

Al factor financiamiento se le puede asignar la siguiente función: "proveer los recursos monetarios para efectuar las inversiones necesarias, así como para desarrollar las operaciones propias de la empresa" (Klein, 1958).

El factor tiene al menos tres elementos, como son: política de financiamiento, la cual debe estar bien definida. Las fuentes para el financiamiento, como patrimonio, créditos bancarios, obligaciones, créditos de proveedores. Los requisitos para lograr esos financiamientos. Los plazos y costos de los fondos, tanto para el aporte del capital propio como para los créditos.
Para diagnosticar este factor se necesita además del cuestionario, conocer varios índices, como:

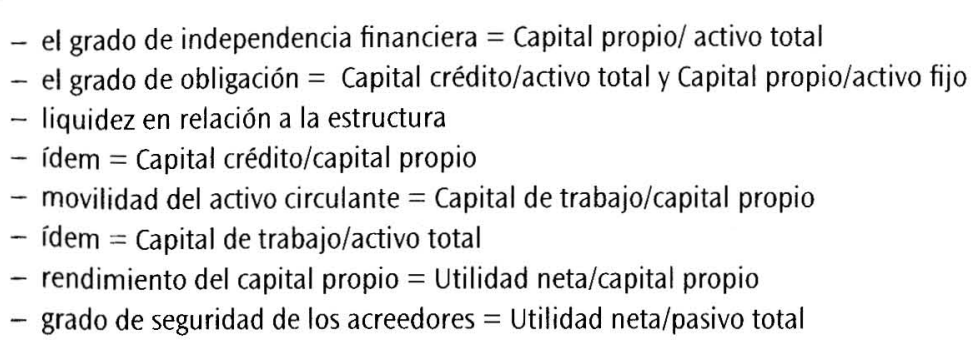

Con el cuestionario es necesario detectar si existe una política consciente para el empleo de los fondos monetarios. ¿Cuáles son las ventajas y desventajas del uso de los fondos? ¿Si se tienen problemas en cuanto a los fondos para la inversión en activos fijos o para el capital de trabajo? ¿Cuál es la incidencia del costo del capital en la operación de la empresa? ¿Cómo se controla la efectividad del uso de fondos? $\mathrm{Y}$ otras preguntas pertinentes.

\section{Medios de producción: el quinto factor para el análisis}

Al factor medios deproducción se le puede asignar la siguiente función: "dotar a la empresa de terrenos, de espacios geográficos, de edificaciones, de maquinarias, equipos y herramientas, de medios de transporte, de servicios locales, etc. que le permitan efectuar sus operaciones con eficiencia" (Klein, 1958).

El factor tiene al menos cuatro elementos, como son: política de inversión y reposición, la cual debe estar bien definida. Servicios prestados por otras empresas o por la misma empresa en materia de transporte, comunicaciones, energía, agua recolección de sólidos, servicios comunales varios. Inversión en suelo (superficie, capacidad, características y valor), en edificaciones (características y distribución según funciones), en maquinarias, equipos y herramientas (clases, valor); y en activos diferidos y capital de trabajo. Además, si se ha definido o no una Política de reposiciones.

Adicional al cuestionario para determinar el cumplimiento de la función asignada a este factor (políticas, problemas, mejoras necesarias, tendencias del mantenimiento y las reposiciones, etc.) se requiere estimar varios índices como: 
intensidad de la inversión = activo fijo/activo total

- intensidad de los gastos y costos fijos = gastos y costos fijos para elaborar los servicios/costo de elaboración total

- grado de mecanización = inversión en maquinarias, equipos y herramientas/activo total - estado del activo fijo = gastos en reparaciones y mantenimiento/activo total

- tasa de inversión relativa a la producción = inversión total/producción física en unidades

\section{Fuerza de trabajo: el sexto factor para el análisis}

Al factor fuerza de trabajo se le puede asignar la siguiente función: "seleccionar, adiestrar y organizar un personal idóneo, tratando de alcanzar la óptima productividad en el desempeño de sus labores" (Klein, 1958).

El factor tiene al menos cinco elementos, como son: política de empleo, que debe estar bien definida. Organización del empleo: reclutamiento, período de inducción, promociones, adiestramiento, rotación. Seguridad y accidentes. Los servicios al personal: alimentos, salud, habitación, transporte, recreación. Personal ocupado por funciones y salarios. Trabajadores calificados y no calificados. Mano de obra directa e indirecta; así como las relaciones laborales o sindicales.

Los índices pueden ser los siguientes:

- relación entre tipos de trabajo $=n^{\circ}$ de empleados $/ n^{\circ}$ de obreros

- relación entre tipos de salarios $=$ salarios directos $/$ salarios indirectos

- horas de trabajo por trabajador $=$ total horas-hombres trabajadas / promedio de trabajadores ocupados

- promedio de remuneraciones por hora $=$ sueldos y salarios totales/ horas-hombres trabajadas importancia de los sueldos y salarios = sueldos y salarios / costo de elaboración de los servicios ventas por trabajador $=$ ventas netas $/ n^{\circ}$ de Trabajadores

- intensidad de la inversión = inversión total $/ n^{\circ}$ de Trabajadores

Las preguntas del cuestionario deben tratar de detectar las reglas para el empleo, la tendencia o no de las renuncias voluntarias, política de adiestramiento, la prevención de accidentes, promedio de trabajadores supervisados por un supervisor, conflictos conocidos y otras preguntas relacionadas con esta función.

\section{Suministros: el séptimo factor para el análisis}

Al factor suministro se le puede asignar la siguiente función: "suministrar a la empresa una corriente continua de materiales y servicios de calidad y a precios convenientes" (Klein, 1958).
El factor tiene al menos cinco elementos, como son: política de compras, la cual debe estar bien definida. La clase, volumen, procedencia de los suministros y sus costos. Los métodos de compra y de existencias. El control de inventarios y organización del almacenamiento; y la Investigación de sustitutos.

Los principales indicadores pueden ser:

- importancia de los abastecimientos = costo de materias primas y materiales/costo general de elaboración

- rotación de los abastecimientos $=$ costo de materias primas y materiales/inventario promedio

- rotación de créditos de proveedor $=$ saldo promedio de créditos de proveedor/costo de abastecimientos de un año

- plazo medio de créditos de proveedor $=360 /$ rotación de crédito de proveedores

El cuestionario debe indagar sobre las normas de compras y la selección de proveedores. Si existen previsiones para posible escasez de materias primas. Si se comparan precios de proveedores y se conocen sus políticas. La relación entre compras y las existencias. Las quejas principales de los proveedores. Las nuevas fuentes de abastecimiento.

\section{Actividad productora: el octavo factor para el análisis}

Al factor actividad productora se le puede asignar la siguiente función: "organizar y efectuar las operaciones de producción en una forma eficiente y económica" (Klein, 1958).

El factor tiene al menos siete elementos, como son: Los métodos de elaboración de los productos y servicios (de acuerdo a pedidos o de acuerdo a existencias). La Organización, planificación y control de los procesos, así como el manejo de materiales según normas. Los turnos de trabajo y el manejo de la temporalidad y el control del uso del tiempo. Los organigramas departamentales. La Capacidad de producción y los costos de producción por unidad vendida. Horas-hombres trabajadas. Las quejas relativas a procesos defectuosos. El uso de nuevas tecnologías.

Algunos de los índices para medir esta ejecución son los siguientes:

- punto de equilibrio en volumen físico de producción

- estructura de los costos $=$ costos fijos/costo de elaboración

- importancia de los gastos de fabricación = costo de elaboración/costo general de producción

- grado de transformación = costos general de producción/costo de materias primas y materiales

- grado de mecanización = consumo de kwh/número de trabajadores

- grado de utilización de la capacidad = producción física/capacidad instalada

- eficiencia de la inspección = producción defectuosa o con quejas/producción total 
El cuestionario para preguntas cualitativas tratará de entender los esfuerzos para mejorar los métodos de producción y si los resultados son o no satisfactorios; así como conocer los costos y sus tendencias, y las horas extra de trabajo; indagar sobre la capacidad utilizada y otras preguntas útiles.

\section{Mercadeo: el noveno factor para el análisis}

Al factor mercadeo se le puede asignar la siguiente función: "adoptar las medidas que garanticen el flujo continuo de los productos y servicios al segmento del mercado objetivo y que proporcionen el óptimo beneficio tanto a la empresa como a los consumidores y la comunidad de acogida" (Klein, 1958).

El factor tiene al menos cuatro elementos, como son: la política de mercadeo bien definida y establecida; los mercados con sus componentes localización, clase y situación de la competencia; las ventas y distribución, con sus componentes organización de las ventas, propaganda y promoción, volumen de ventas, precios y condiciones, supervisión de ventas y sistemas de distribución; luego el elemento investigación de los mercados.

Los índices que más se utilizan para este diagnóstico son:

- punto de equilibrio sólo de la operación de mercadeo

- importancia de lo vendido = gastos de ventas y distribución/costo de lo vendido

- influencia de la localización = gasto de transporte/costo de lo vendido

- influencia de la propaganda y promoción = costo de propaganda y promoción/ventas netas

- venta por vendedor $=$ ventas netas/número de vendedores o de guías

- importancia de la investigación de mercado = costo de la investigación de mercados/ventas netas

El cuestionario trata de obtener datos sobre la clientela que se espera, los precios y las condiciones, las posibilidades de mejorar las ventas y qué se hace al respecto, precios de los productos y servicios similares competidores, fortalezas y debilidades frente a la competencia, aumento o no de la clientela, ascenso o descenso de los precios; previsiones de ventas e investigaciones.

\section{Contabilidad y estadística: el décimo factor para el análisis}

Al factor contabilidad y estadística se le puede asignar la siguiente función: "establecer y tener en funcionamiento una organización para la recopilación de datos, particularmente de mercados, financieros y de costos, con el fin de man- tener informada a la empresa de los aspectos económicos de sus operaciones" (Klein, 1958).

El factor tiene al menos tres elementos, como son: la organización contable con sus elementos presupuesto, contabilidad financiera, registros de ventas y costos; los informes contables, o sea balance, estado de resultado, flujo de Caja, informes de Producción, de ventas y costos; y las auditorias continuas o intermitentes, interna y externa.

Las principales fórmulas para medir esta ejecución son:

- rendimiento de ventas $=$ utilidad neta/ventas netas

- rendimiento de activos totales $=$ utilidad neta/activos totales

- rendimiento de activos fijos = utilidad neta/activos fijos

- rentabilidad financiera temporal $=$ flujo de caja descontado (valor actual neto y tasa interna de retorno)

El cuestionario sobre el cumplimiento de las funciones trata de hacer preguntas para indagar sobre el éxito y los problemas de la organización contable en cuanto a número de personas, métodos y recursos financieros asignados. La oportunidad de los informes y su uso efectivo o no. Los Informes periódicos sobre costos fijos y variables, modos de hacer las auditorias y sus responsables.

\section{La importancia del diagnóstico}

La empresa turística es la célula más pequeña del sistema turístico; y como tal, forma parte de un conjunto que tiene entre todos sus propias vinculaciones. La marcha dinámica de estas empresas se manifiesta en una continua mejora de sus productos y servicios e incluso de sus técnicas de elaboración, para asegurar sus mercados frente a la competencia. Igualmente, se debe determinar, a tiempo, qué actividades se apartan de la tendencia, a fin de poder facilitar las correcciones para no caer en situaciones difíciles.

Los diagnósticos son útiles porque se puede disponer de información adecuada y oportuna, para poder tomar decisiones o cambiar las políticas. Es necesario conocer las causas de las dificultades y prever lo que pueda suceder, como una base para establecer estrategias. Se necesita interpretar los parámetros operativos, una vez que se determinan los factores, sus funciones y sus elementos. Aquí nos hemos concentrado en sólo diez factores, y para cada uno hemos definido una función en relación al objetivo general de la empresa; y por lógica, el diagnóstico tratará de determinar el grado en que el desempeño real y objetivo de estas funciones contribuye al esfuerzo de la empresa como un sistema integrado. Si logramos co- 
nocer: ¿cuáles son los factores que limitan en forma adversa la operación total de la empresa turística?, estaríamos en una adecuada posición para hacer correcciones a tiempo. De allí la importancia del diagnóstico.

\section{Apreciaciones y juicios subjetivos}

La investigación de empresas parece estar mucho más cerca, por la complejidad de sus factores, de las ciencias biológicas que de las ciencias físicas; de allí que sólo se pueda avanzar si la investigación cuantitativa se complementa con la cualitativa.

La observación de los problemas tiene que ser integral. El problema consiste en obtener datos duros. ¿Qué quiere decir esto?: que a menudo puede suceder que se parta de una hipótesis más simplista; y sin embargo, por no profundizar en el asunto, se puede obtener resultados errados.

También es necesario advertir que cuando se hacen preguntas a personas experimentadas, existe también el peligro de obtener respuestas inconscientemente predispuestas, pues ya se sabe que las opiniones personales de eventos fortuitos, a veces no son las correctas, pues se tiene la tendencia a conservar en la mente representaciones que han producido una profunda impresión; y ello hace que se olviden otros asuntos y hechos. De allí que sólo relacionando, en forma sistemática, el diagnóstico de todos los factores, podríamos liberar hipótesis mucho más relevantes y válidas.

El diagnóstico y su metodología sólo es una herramienta de las tantas que se pueden usar y que puede facilitar el trabajo de una manera más ordenada. Siempre el asunto importante es poder obtener, de fuentes confiables, datos y hechos útiles para el análisis.

Dijimos que este diagnóstico esta más cerca de la biología que de la física. Pues bien, como la empresa es la célula más pequeña del cuerpo económico de una sociedad, entonces no es de extrañar que el cuerpo económico mismo esté luchando para satisfacer los deseos y necesidades originados por sus procesos y por los individuos que participan en la actividad económica; por esto, la función de una empresa consiste, por tanto, en contribuir a la satisfacción de esas necesidades; de allí que la empresa, además de obtener ganancias, sea un establecimiento subordinado a los intereses sociales de una comunidad. Por ejemplo, la empresa turística debe rendir un servicio adecuado para la rama a la cual pertenece, tomando en consideración la comunidad; y para el sector turístico en su totalidad, pues de otra manera puede ser expulsada del cuerpo económico y perecer como una célula sin función alguna.
Por lo que se refiere a las actividades de las empresas, en realidad ellas están allí para vender el tiempo de trabajo de todos sus empleados y la depreciación de sus bienes de producción, transformando las materias primas y los servicios comprados, en nuevos productos y servicios que serán consumidos por una clientela; pero también la empresa debe ayudar para que la comunidad, donde esté inserta, pueda prosperar con ella. El mejor empleo de los insumos, se debe traducir en un incremento de la productividad. La combinación óptima de los recursos disponibles, debe ser la base para proporcionar buenos servicios; pero durante su actividad pueden surgir fallas, que por lo general provienen de los diez factores que hemos mencionado con anterioridad, pues estos factores de operación sirven de base para el diagnóstico.

Digamos entonces que los factores son los constituyentes anatómicos de las operaciones de la empresa, y las funciones o actividades relacionadas con ellos, la fisiología o el desempeño de la misma. Si todos los desempeños asignados no fallan, el cuerpo económico de la empresa estará sano. No obstante, las funciones mal desempeñadas dan lugar a ineficiencias de la empresa, por lo que es necesario lograr el equilibrio de todos sus factores y sus funciones.

Por supuesto, las funciones difieren en importancia o "peso", de acuerdo con su relativa contribución al total; y además, estos pesos pueden variar según tipos específicos de empresas y ramas de negocios; e incluso, si se toman en cuenta consideraciones de corto, mediano o largo plazo.

El peso de cada función desempeñada, o sea: la intensidad con que se perciben sus efectos, son diferentes según las condiciones operativas. Con todo, se puede aplicar la Ley del mínimo de Liebig, químico y biólogo quien señaló en el siglo pasado que: "la deficiencia o ausencia de un elemento necesario, aún cuando existan todos los demás, produce esterilidad en el suelo, si se trata de cultivos para cuya vida sea indispensable dicho elemento". En resumen, una función de operación desempeñada con poca eficiencia, limita el rendimiento del conjunto de operaciones. Es necesario conocer las funciones deficientes para adecuarlas y mejorar le operación total.

El incremento, en forma aislada, de un factor operativo no tiene razón de ser, pues sería un gasto de energía inútil, pues lo importante es el conjunto de esfuerzos "moleculares", ya que a veces los elementos o componentes de un factor, pueden ser los que han fallado y ello supone desagregar el análisis. La complejidad de las fuerzas interrelacionadas e interdependientes hace difícil el intento de formular el problema solamente con indicadores cuantitativos, pues las normas de cumplimiento pueden estar apoyadas en otros elementos, que al ser cualitativos, pueden contribuir al logro de un desempeño equilibrado. 


\section{Una manera de valorar}

Como se sabe, la productividad consiste en comparar las cantidades físicas de un producto contra los insumos o recursos que se necesitan para obtenerlo. Por ejemplo, la productividad del trabajo es igual a: cantidad física de producto/horas-hombres trabajadas. Este índice de productividad aumenta con el incremento de cualquier componente del producto; o bien, con la disminución de cualquier componente del insumo. Por ello cada factor, formado por sus elementos, puede contribuir al aumento de la productividad, que es el resultado combinado del cumplimiento de las funciones. Y si surge una función menos eficiente, esto quiere decir que ella ha sido responsable en disminuir el producto o bien aumentar los insumos o viceversa. Así pues, si existen diez factores y cuatro posibilidades (aumento y disminución del producto y del insumo), entonces serán cuarenta grupos de variables que pueden alterar la productividad de una empresa.

Una vez que se ha diagnosticado cada factor con sus elementos, es necesario determinar el peso o importancia de cada uno, según el marco referencial que corresponda, para establecer la posible contribución de cada factor en la productividad total.

La valoración de cada factor, considerando sus elementos, se puede catalogar de: $\mathrm{a}=$ satisfactoria; $\mathrm{b}=$ limitada; $\mathrm{y} \mathrm{c}=$ inadecuada. La fórmula para conocer la efectividad del factor puede ser como sigue: en el numerador aparece la sumatoria de los elementos satisfactorios multiplicado por la unidad; la sumatoria de los elementos limitados multiplicados por $1 / 2$; y finalmente, la sumatoria de los elementos inadecuados multiplicados por $1 / 4$. En el denominador el número total de elementos valorados.

Supongamos que estamos valorando el factor Productos y Procesos que lo hemos tabulado de la siguiente manera:

\begin{tabular}{|c|c|c|c|}
\hline Elementos del factor & & & \\
\hline & a & b & c \\
\hline Política empleada & & $x$ & \\
\hline Clase de producto (servicios) & & $\mathrm{x}$ & \\
\hline Métodos de producir el servicio: & & & \\
\hline - características de sus componentes & & $\mathrm{x}$ & \\
\hline - características de su organización & & & $\mathrm{x}$ \\
\hline - características de las mejoras & & & $\mathrm{x}$ \\
\hline$N=5$ & 0 & 3 & 2 \\
\hline
\end{tabular}

Efectividad $=(0 / 1+3 / 2+2 / 4) / 5=2 / 5=0.40=40 \%$ de efectividad
Supongamos que después de valorarse todos los diez factores, obtenemos las siguientes efectividades:

\begin{tabular}{|l|l|}
\hline 1. Medio ambiente & $54 \%$ \\
\hline 2. Política y dirección & $54 \%$ \\
\hline 3. Productos y procesos & $40 \%$ \\
\hline 4. Financiamiento & $75 \%$ \\
\hline 5. Medios de producción & $45 \%$ \\
\hline 6. Fuerza de trabajo & $55 \%$ \\
\hline 7. Suministros & $78 \%$ \\
\hline 8. Actividad productora & $39 \%$ \\
\hline 9. Mercadeo & $56 \%$ \\
\hline 10. Contabilidad y estadística & $38 \%$ \\
\hline
\end{tabular}

El máximo de efectividad sería 100\% y el mínimo 25\% (mínimo para subsistir). Los valores comprendidos entre $40 \%$ y $60 \%$ son valores medios. Así pues, en la ilustración del ejemplo anterior, los tres principales factores limitantes son: contabilidad y estadística, actividad productora y productos-procesos $(38 \%, 39 \%$ y 40\%); luego los segundos tres factores limitante son: medios de producción, medio ambiente y política-dirección $(45 \%, 54 \%$ y $54 \%)$; y los cuatro factores restantes son: fuerza de trabajo, mercadeo, financiamiento y suministros (55\%, $56 \%, 75 \% 78 \%$ ).

Ahora bien, en este ejemplo podemos decir que la clasificación para ordenar tentativamente a los factores es como sigue: suministros 1; financiamiento 2; mercadeo 3; fuerza de trabajo 4; medio ambiente 5; política y dirección 5; medios de producción 6 ; productos y procesos 7 ; actividad productora 8 ; contabilidad y estadística 9.

En seguida se debe otorgar un peso o importancia a cada factor clasificado. Este asunto de otorgar importancia depende de la naturaleza de la empresa que se evalúa. Supongamos que se considera una empresa pequeña que no requiere un elevado financiamiento, pero que si necesita una fuerza de trabajo eficiente y unos productos y procesos bien definidos; y además, medios de producción oportunos. Supongamos que los suministros de espacios geográficos, para su desempeño, están asegurados y que debido a sus recursos prístinos y a la cultura de la comunidad de acogida se asegura relativamente el éxito para sus mercados; pero que también existe mucha competencia en el ambiente por este tipo de servicios, por lo que la política y dirección trabajaría bajo incertidumbre y riesgo; y así, la actividad productora se debe realizar con el análisis de una buena data contable y de estadísticas, a fin de contratar buenos canales de mercadeo. 
Según el marco de circunstancias anteriores, los pesos otorgados para determinar la contribución de cada factor al total sería como sigue: financiamiento $3 \%$; fuerza de trabajo $7 \%$; productos y procesos $8 \%$; suministros $8 \%$; ambiente $8 \%$; política y dirección $10 \%$; actividad productora $10 \%$; medios de producción $11 \%$; contabilidad y estadística 19\%; mercadeo, 16\%. (La suma es igual al 100\%).

Si se aceptaran esos pesos como probables, entonces el modelo para tabular y combinar los hallazgos de esta empresa aparece en seguida:

\begin{tabular}{|l|c|c|c|c|c|}
\hline Factor & Rango & \% en peso & Efectividad & b xc/ 100 & Áreas Críticas \\
\hline Ambiente & 5 & $8 \%$ & $54 \%$ & 4,32 & \\
\hline Política y dirección & 5 & $10 \%$ & $54 \%$ & 5,40 & \\
\hline Productos y proc. & 7 & $8 \%$ & $40 \%$ & 3.20 & $\mathrm{X}$ \\
\hline Financiamiento & 2 & $3 \%$ & $75 \%$ & 2.25 & $\mathrm{X}$ \\
\hline Medios de prod. & 6 & $11 \%$ & $45 \%$ & 4.95 & \\
\hline Fuerza de trabajo & 4 & $7 \%$ & $55 \%$ & 3.85 & $\mathrm{X}$ \\
\hline Suministros & 1 & $8 \%$ & $78 \%$ & 6,24 & \\
\hline Actividad prod. & 8 & $10 \%$ & $39 \%$ & 3.90 & $\mathrm{X}$ \\
\hline Mercadeo & 3 & $16 \%$ & $56 \%$ & 8.96 & \\
\hline Contabilidad & 9 & $19 \%$ & $38 \%$ & 7.22 & \\
\hline & & $100 \%$ & & 50.29 & \\
\hline
\end{tabular}

Del resultado total del diagnóstico, los factores críticos que merecen ser discutidos con todos los interesados involucrados con esta empresa, incluida la comunidad de acogida, son: los productos y procesos; financiamiento; la fuerza de trabajo, que es clave; y la actividad productora, la cual se ha visto impactada. Pareciera entonces, que la operación actual de la empresa requiere de perfeccionamientos inmediatos para elevar la producción y bajar los costos. Esto debería ir acompañado de un adiestramiento de la fuerza de trabajo y de una mejor organización de las tareas, de manera que permita una actividad productora eficiente.

En la Figura 2 el grado de efectividad aparece sin aplicar todavía las ponderaciones. Al otorgar los pesos que corresponden, el factor financiamiento al recibir un bajo porcentaje sobre el total, debido a la naturaleza de la empresa y su tamaño, aparece como un área critica.

\section{Grado de cumplimiento de los factores}

Factores

1 - Medio ambiente

2 - Política y dirección

3 - Productos y proceso

4 - Financiamiento

5 - Medios de producción

6 - Fuerza de trabajo

7 - Abastecimientos

8 - Actividad productora

9 - Mercadeo

10 - Contabilidad y Estadística

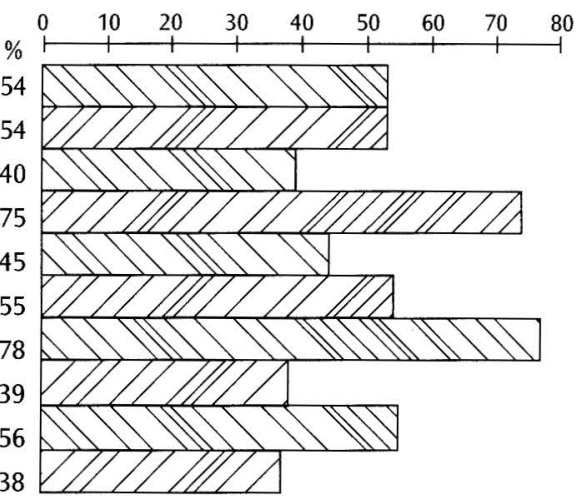

Figura 2. Gráfico - Grado de cumplimiento de los factores (efectividad sin ponderación)

\section{Factores para el diagnóstico macroeconómico}

Una manera práctica de realizar los diagnósticos en relación a la importancia de un sector de la economía o de una actividad económica muy específica, consiste en utilizar metodologías más sofisticadas como la herramienta denominada matriz de Insumo-Producto, desarrollada hace muchos años por Wassily Leontief, Premio Nóbel de Economía de 1973.

Nos decía Hollis B. Chenery (1970) sobre esa herramienta de análisis lo siguiente:

El análisis input-output ha probado ser una de las más ricas innovaciones para la economía aplicada. Este modelo ha sido construido por más de 50 países y aplicado a centenares de problemas específicos. Aunque el debate continúa en relación a los méritos acerca de las alternativas teóricas para la búsqueda del equilibrio general del sistema, el valor práctico del enfoque input-output está mucho más allá de ser cuestionado.

Es decir, el diagnóstico endógeno sobre una actividad económica cualquiera, se puede complementar con el diagnóstico de su interdependencia con otras iniciativas económicas, pues todas tratan de asignar mejor sus recursos disponibles.

Los cambios estructurales en los países emergentes son muy necesarios, pero estos cambios no se pueden realizar abandonando las técnicas de planificación.

La técnica del insumo-producto comenzó a considerarse como potencialmente útil sólo en el año de 1968, aunque ya se había discutido sobre ella en el 
año de 1953, pero sólo como un asunto especulativo y teórico. Especialmente sus aplicaciones en el campo de las políticas ínter industriales han sido numerosas, donde se buscaban las prioridades sociales a través de la planificación y políticas públicas. Los análisis se enfocaron también en sectores particulares de la economía, como por ejemplo las tablas i-o de la Economía Turística Española de los años 70 (Figuerola, 1976), que en realidad fue una manera de diagnosticar sus problemas relativos al comercio internacional de los viajes; por eso, este modelo ha sido diseñado para un propósito específico, el cual sirvió para conocer las posible políticas alternativas en materia de turismo.

Para buscar un esquema que permitiera la desagregación del turismo se dijo que:

- el turismo lleva consigo una estrecha relación con el transporte;

- el turismo utiliza medios de alojamiento;

- el turismo se sirve de agentes intermediarios;

- el turismo se relaciona en forma directa con una planta recreativa;

- el turismo consume un amplio abanico de productos y servicios.

Veamos en seguida una definición casi completa sobre el fenómeno turístico que aportó en España el economista José Largo Jiménez (1976):

Desde el punto de vista económico, se considera turismo toda actividad que supone para el sujeto un consumo de bienes y servicios, localizado en un lugar distinto al que corresponde a su residencia habitual. Esta actividad de consumo, previamente exige desplazamiento de la persona a un lugar fuera de su residencia habitual, cualquiera que sea el motivo de su viaje. El gasto de consumo se materializa en una gran variedad de bienes y servicios producidos por las empresas. El "producto turístico" no posee homogeneidad en su propia naturaleza; son las características del mismo y las circunstancias en que se adquiere las que lo determinan. Las empresas oferentes, por lo tanto, se agrupan en distintos sectores en la agregación clásica por productos homogéneos, aunque determinadas actividades pueden considerarse como puramente turísticas.

\section{El consumo turistico}

Se trata de un consumo que se realiza fuera del domicilio habitual. El consumo se puede clasificar en: consumo turístico de unidades familiares o sea la demanda final; consumo turístico de las empresas o inputs intermedios, que son costos de producción para las empresas; el consumo turístico del gobierno que igualmente son inputs intermedios o costos para el gobierno; el consumo turístico exterior, que corresponde al consumo del turismo receptivo o internacional en el país en cuestión y los servicios turísticos que provienen del exterior.

La sumatoria de todos estos consumos se considera la demanda total realizada in-situ; la cual a su vez, se clasifica en consumo del turismo doméstico y el consumo del turismo internacional

\section{La producción turística}

No parece muy clara la delimitación del turismo del lado de la oferta, pues el carácter de los bienes y servicios producidos por otros sectores no turísticos depende del destino final. Por esto, se puede distinguir dos grandes grupos de sectores productivos: las empresas que pueden considerarse puramente turísticas, debido a que sus servicios siempre van dirigidos a un visitante temporal que realiza un consumo turístico, como: agentes y operadores de viajes, transporte para viajeros y servicios de alojamiento a clientela no estable; en cambio los servicios de alimentos y bebidas son más difíciles de catalogar como turísticos, pues ellos sirven también a personas que tienen su residencia habitual en el mismo lugar de su ubicación. Existen otras empresas que igualmente pueden servir a los visitantes temporales, pero también a los residentes en el sitio.

\section{La inversión turística}

Para producir los servicios turísticos se necesitan inversiones y siempre para este rubro se consideran solamente las que se relacionan directamente con actividades propiamente turísticas, es decir las inversiones en instalaciones para el alojamiento, medios de transporte utilizados por visitante temporales y las inversiones realizadas por operadores y agentes de viajes, así como las obras de infraestructura de carácter eminentemente turístico.

\section{Algunos hallazgos importantes que permitieron fijar políticas}

No hay duda que al contar con una matriz Insumo-Producto se puede realizar un diagnóstico que permita conocer determinadas relaciones y la importancia de determinados servicios. Por ejemplo, en el caso del turismo español, el $28 \%$ de los gastos relativos al consumo total del turismo doméstico se realizan en otras actividades no calificadas como turísticas. 
En cuanto al turismo internacional este mismo porcentaje es igual al 38\% especialmente en alimentos, compras en negocios comerciales y explotación de bienes inmuebles. En seguida aparece una comparación porcentual entre el consumo turístico realizado por el visitante doméstico y el consumo turístico realizado por el visitante internacional.

La estructura del gasto turístico total, tanto del turismo doméstico como del turismo internacional, es como sigue:

\begin{tabular}{|l|c|}
\hline Servicio de operadores y agentes de viajes & $1,0 \%$ \\
\hline Servicio de transporte & $12,3 \%$ \\
\hline Servicio de alojamiento turístico y recreación & $59,4 \%$ \\
\hline Otras actividades no turísticas & $27,3 \%$ \\
\hline Total & $100 \%$ \\
\hline
\end{tabular}

Del rubro alojamiento y recreación, el alojamiento propiamente dicho representa el $27 \%$, la alimentación y bebidas en restaurantes, cafeterías, bares, tabernas el $60 \%$ y la recreación realizada en salas de fiesta, casinos, balnearios y campamentos es igual al $13 \%$

Si se examina en detalle los servicios por tipología de turismo, los resultados aparecen como sigue:

\begin{tabular}{|l|c|c|}
\hline & Doméstico & Internacional \\
\hline Servicios de transporte terrestre, marítimo y aéreo & $15,04 \%$ & $13,26 \%$ \\
\hline Servicio de operadores y agentes de viajes & 0,40 & 0,60 \\
\hline Servicio de alojamiento y recreación & 57,61 & 60,14 \\
\hline Otras actividades turísticas & 26,95 & 26,00 \\
\hline
\end{tabular}

Del consumo total el 65\% lo realizan las unidades domésticas y el 35\% restante el visitante internacional. Igualmente, el consumo se puede dividir en consumo realizado en actividades propiamente turísticas $(73 \%)$ y el consumo realizado en otras actividades (27\%); es decir, la exportación in-situ del turismo corresponde al 35\% del gasto del consumo turístico, o lo que es lo mismo: se suponen que en ese porcentaje existe un ingreso expresado en divisas.

Los rubros turísticos que más se "exportan" del consumo total son: del total de alojamiento turístico y actividades recreativas (45\% corresponde a turismo internacional), del consumo total de transporte, especialmente el aéreo (38\% corresponde al turismo internacional); y finalmente, del consumo total de bienes y servicios comerciales que no son propiamente turísticos (63\% corresponde al turismo internacional).

Se estima que el porcentaje del contenido de importaciones en consumo turístico total es más o menos del $4 \%$ en actividades turísticas y del $8 \%$ en actividades no turísticas.

En el consumo turístico la proporción de valor agregado bruto es cerca del $52 \%$. El alto contenido de sueldos y salarios de las actividades turísticas, es una demostración más que está actividad de servicios puede aliviar, en parte, los problemas del desempleo de mano de obra.

De la actividad turística total (consumo más inversión) el $81 \%$ es consumo turístico y el 19\% restante es inversión turística en planta y en medios de transporte. Del consumo total el 59\% lo producen los servicios de alojamiento y recreación.

Este diagnóstico se puede realizar en España porque el país cuenta con una Cuenta Satélite de Turismo dentro de los datos que aporta su Banco Central.

\section{Consideraciones finales}

El diagnóstico y la interpretación, aunque se pueden distinguir conceptualmente, por lo general aparecen unidos en la práctica. Se puede decir que el diagnóstico reclama la interpretación y ambos constituyen pasos sucesivos de un mismo proceso (Sierra Bravo, 1981). El punto de partida del análisis es, desde luego, la existencia de un problema o una situación que requiere alguna solución.

El objetivo principal del método presentado para realizar un diagnóstico macro o microeconómico, consiste en describir las causas de una baja productividady, conociéndolas, establecer las bases para aumentarla. En resumen, el método es un diagnóstico de la productividad. La labor analítica comenzó con la determinación de diez factores y sus funciones; factores que intervienen en la operación de una empresa. Luego se recopilaría la información cuantitativa y cualitativa sobre cada uno de esos factores y sus elementos. Se usan mediciones de efectividad, con el objeto de descubrir las áreas en donde se deben hacer mejoras; y luego, asignando pesos se puede llegar a interpretar las áreas críticas para tomar decisiones.

Por su parte, el objetivo principal del diagnóstico macroeconómico consiste en buscar una herramienta de análisis que permita conocer las características del consumo turístico, la producción y la inversión turística, tanto relacionada con el turismo doméstico como con el turismo internacional. La finalidad de este análisis es poder realizar una adecuada planificación del sector y establecer criterios de políticas, según los hallazgos encontrados. 


\section{Referencias bibliográficas}

BARRETTO, Margarita. 2001. Manual de iniciação ao estudo do turismo. Campinas, SP: Papirus.

BENI, Mário C. 2002. Análisis estrutural do turismo. 7.ed. Sao Paulo: SENAC.

BRENT R. J. R. \& GOELDNER, C. (Eds.). 1987. Travel, tourism, and hospitality researchers: a handbook for managers and researchers. New York: John Wiley \& Sons.

BRIONES, Guillermo. 1982. Métodos y técnicas de investigación para las ciencias sociales. México: Trillas.

CARTER, A. P. \& BRODY, A. (Eds.). 1970. Aplications of input-output analysis. Ámsterdam: North-Holland Publishing Company.

FIGUEROLA, Manuel. 1995. Economía para la gestión de las empresas turísticas. Madrid: Síntesis.

1990. Elementos para el estudio de la economía de la empresa turística. Madrid: Estudios Ramón Areces.

1976. Las tablas de i-o de la economía turística española. Estudios Turísticos, n. 49-50. Separata.

KLEIN, Alfred. 1966. El diagnóstico de empresas. Caracas: Arte.

1961. La productividad como criterio de inversión: guía para evaluación de proyectos industriales. México: Morales Hnos.

. \& GRABINSKY, N. 1958. El análisis factorial: una guía para estudios de economía industrial. México: Victoria.

LARGO JIMENEZ, José. 1976. El turismo y el mercado interior en España. Estudios Turísticos, n. 49-50, enero-junio, Madrid.

REJOWSKI, Mirian.1996. Turismo e pesquisa cientifica. Campinas, SP: Papirus.

SCHLÜTER, Regina. 2000. Investigación en turismo y hotelería. Buenos Aires: CIET.

SMITH, S. L. J. 1989. Tourism analysis: a handbook. New York: Longman Scientific \& Technical.

SIERRA BRAVO, Restituto. 1981. Ciencias sociales: análisis estadístico y modelos matemáticos. Madrid: Paraninfo.

Recibido en: 02/07/05.

Aprobado en: 16/07/05. 